\title{
Toxic Erythema of Chemotherapy
}

National Cancer Institute

\section{Source}

National Cancer Institute. Toxic Erythema of Chemotherapy. NCI Thesaurus. Code C112182.

Painful red or purple patches, edematous plaques or blisters on the hands, feet, extremities or intertriginous regions; the onset can occur days to months after receiving chemotherapy. 